\title{
Revista Colombiana de

\section{Capítulo 12. Utilidad del mapeo tridimensional en la ablación de taquicardia ventricular en cardiopatía chagásica}

\section{Chapter 12. Usefulness of three-dimensional mapping in ventricular tachycardia ablation in Chagas cardiomyopathy}

\author{
Guillermo Mora ${ }^{\mathrm{a}, \mathrm{b}, \mathrm{c}, *}$, Miguel A. Vacca ${ }^{\mathrm{c}, \mathrm{d}}$ y Carlos A. Sánchez
}

a Universidad Nacional de Colombia, Bogotá, Colombia

' Fundación Santafé de Bogotá, Bogotá, Colombia

c Hospital Universitario Clínica San Rafael, Bogotá, Colombia

' Fundación Clínica Shaio, Bogotá, Colombia

Recibido el 16 de noviembre de 2015; aceptado el 18 de enero de 2016

\section{Introducción}

La enfermedad de Chagas fue descrita en 1909 por Carlos Chagas y es en la actualidad la tercera entidad parasitaria más frecuente en el mundo ${ }^{1}$. Un estudio realizado en 2006 concluyó que 7,6 millones de personas se encuentran infectadas en Latinoamérica ${ }^{2}$ y se cree que hay 38.000 nuevos casos cada año ${ }^{3}$. En Colombia, según datos del Instituto nacional de salud, existen entre 700.000 y 1.200 .000 habitantes infectados, y se estima que 8 millones de personas están en riesgo de adquirir la infección ${ }^{4}$. Así pues, esta enfermedad representa un problema de salud pública en varios países de la región, ya que es la principal causa de miocardiopatía no isquémica en el continente americano ${ }^{5}$.

Entre los pacientes que adquieren la infección, la complicación más frecuente y peligrosa es la miocardiopatía chagá- sica, la cual se desarrolla en el 25 al $40 \%$ de los casos y suele manifestarse cuando han transcurrido 20 o 30 años del contacto con el parásito ${ }^{3}$. La miocardiopatía chagásica es una forma de miocarditis crónica que compromete las cámaras cardiacas, los sistemas simpático y parasimpático y de conducción ${ }^{6}$. Se presenta en tres formas clínicas que pueden o no coexistir en un mismo paciente: arritmias cardiacas, falla cardiaca y tromboembolia pulmonar y sistémica7.

El desarrollo de la miocardiopatía tiene implicaciones pronósticas significativas. Un estudio reciente mostró que los pacientes con esta condición tienen mayor riesgo de muerte prematura y que la mortalidad por cualquier causa es cuatro veces mayor que en pacientes con falla cardiaca por otra etiología ${ }^{8}$. Una de las causas que explica este aumento en la mortalidad es el desarrollo de arritmias potencialmente fatales, entre las cuales cabe mencionar el rol de la taquicardia ventricular?. 


\section{Taquicardia ventricular en cardiopatía chagásica}

Los mecanismos fisiopatológicos que conllevan al desarrollo de arritmias cardiacas en el paciente con cardiopatía chagásica, aún no son claros. Estudios patológicos en pacientes con muerte súbita han descrito la presencia de disautonomía chagásica, caracterizada por denervación simpática y parasimpática ${ }^{10}$. La pérdida de la regulación autonómica sobre el corazón predispone al paciente al desarrollo de arritmias ventriculares, incluso en corazones con fracción de eyección conservada ${ }^{11}$. La prevalencia y severidad de las arritmias ventriculares se relaciona de manera directa con la gravedad de la miocardiopatía, pero pueden darse en pacientes sin disfunción ventricular ${ }^{12}$.

De esta manera, se sabe que la prevalencia de taquicardia ventricular no sostenida es del $40 \%$ en pacientes con trastornos de contractilidad documentados y del $90 \%$ en pacientes con falla cardiaca instaurada. La prevalencia de taquicardia ventricular sostenida es menor pero implica un mal pronóstico, no sólo por ser la principal causa de muerte súbita en pacientes con Chagas, sino porque involucra mayor riesgo de progresión de la enfermedad ${ }^{13}$.

La muerte súbita constituye la principal causa de muerte en estos pacientes, con valores cercanos al $40 \%$ en algunos estudios $^{14}$. La mayoría ocurre en pacientes con cardiopatía clínicamente manifiesta, entre los 30 y 50 años de edad. Sin embargo, hasta un $20 \%$ de los casos de muerte súbita ocurre en ausencia de síntomas previos ${ }^{15}$.

El manejo de las arritmias ventriculares no sostenidas es expectante ya que su tratamiento no cambia el pronóstico del paciente. El uso de antiarrítmicos en pacientes con este tipo de arritmias y disfunción ventricular izquierda es controvertido, pero un metaanálisis no demostró cambios en la mortalidad total con el uso de amiodarona, si bien no fue dirigido a pacientes con cardiopatía chagásica ${ }^{16}$. En pacientes sintomáticos con arritmias ventriculares no sostenidas y disfunción ventricular, la amiodarona produce mejoría de los síntomas mas no así un cambio importante en el pronóstico ${ }^{17}$.

En pacientes recuperados por muerte súbita asociada con arritmias ventriculares malignas, el manejo de elección es el implante de un cardiodesfibrilador (CDI). Esta estrategia se deriva de estudios que no incluyeron pacientes con enfermedad de Chagas, pero es de amplia aceptación por la comunidad médica ${ }^{17}$. Estudios observacionales sugieren que los pacientes con cardiopatía chagásica tienen mayor número de eventos de arritmias ventriculares así como de choques, aunque sin cambios en la mortalidad ${ }^{18}$. Un estudio publicado en 2014 por Gali et al. ${ }^{19}$ comparó el implante de CDI más amiodarona vs. amiodarona sola, en pacientes con arritmias ventriculares y cardiopatía chagásica, y concluyó que aquellos tratados con dispositivo tuvieron menor riesgo de mortalidad por todas las causas así como de muerte súbita, y que dicho beneficio fue mayor en pacientes con fracción de eyección menor al 40\%. En nuestra población un estudio encontró que casi el $20 \%$ de los pacientes con dispositivos implantados (marcapasos o cardiodesfibriladores) tenían cardiopatía de origen chagásico ${ }^{20}$. La utilidad en prevención primaria es menos estudiada.

En la medida en que los pacientes con Chagas tengan mayor frecuencia de descargas, estas podrán ser muy dolorosas y algunos llegarán a quejarse de un deterioro en su calidad de vida ${ }^{21}$. Por tanto se requieren estrategias para disminuir los episodios de taquicardia ventricular.

\section{Utilidad del mapeo tridimensional}

Se ha establecido que el principal mecanismo de taquicardia ventricular en estos pacientes es el desarrollo de circuitos de reentrada en cicatrices fibróticas, el $70 \%$ de las cuales se encuentra en la pared inferolateral del ventrículo izquierdo $^{22}$. Prevenir la taquicardia ventricular mediante ablación disminuye la carga de terapia con desfibrilador y potencialmente mejora la calidad de vida. Por ello, la ablación surgió como una opción terapéutica de gran valor. Adicionalmente, un estudio publicado en 2002 demostró que ésta disminuye la mortalidad por falla cardiaca en este grupo de pacientes ${ }^{23}$.

En general, las indicaciones de ablación en pacientes con taquicardia ventricular son las siguientes:

- Control de taquicardia ventricular monomórfica sintomática sostenida que recurre a pesar de tratamiento antiarrítmico.

- Intolerancia al tratamiento antiarrítmico.

- Control de taquicardia ventricular incesante.

El éxito de la ablación depende de la identificación exacta del foco arritmogénico. Algunos pacientes con cardiopatía chagásica tienen extensas áreas de fibrosis, que generan múltiples focos de arritmia. En estos pacientes se requiere un mapeo extenso para lograr una ablación exitosa ${ }^{24}$.

Una situación que hace más complejo el manejo de estos pacientes, es que los focos arritmogénicos pueden estar localizados en fibras subendocárdicas, intramiocárdicas o subepicárdicas. En algunos pacientes puede lograrse la ablación por catéter de manera exitosa, sin embargo, en muchos de ellos el foco se encuentra completamente intramiocárdico o en una localización subepicárdica que dificulta notoriamente la ablación exitosa ${ }^{25}$.

La meta de la ablación es identificar istmos críticos para el mantenimiento de la taquicardia y eliminarlos mediante radiofrecuencia. No es raro encontrar varias morfologías de taquicardia ventricular que necesitan procedimientos con mapeos minuciosos. Una característica importante de los pacientes con taquicardia ventricular y cardiopatía chagásica es que hasta un 30 a un $50 \%$ de las taquicardias ventriculares, tienen origen en circuitos epicárdicos (fig. 1) ${ }^{26}$.

La aproximación epicárdica se realiza por punción subxifoidea, con bajas complicaciones mayores (1-2\%) ${ }^{27}$. Las señales electrográficas del epicardio se asemejan a las del endocardio; sin embargo, al realizar la ablación puede producirse lesión de las arterias coronarias o de tejidos circundantes como el nervio frénico ${ }^{28}$.

El mapeo electroanatómico es fundamental para definir claramente los circuitos de la o las taquicardias ventriculares. En general, se ha considerado que los electrogramas bipolares con voltaje $>1,5 \mathrm{mV}$ son tejido normal, voltajes $<0,5$ $\mathrm{mV}$ representan cicatriz eléctrica y entre 0,5 y $1,5 \mathrm{mV}$ una zona de transición entre el tejido sano y el cicatricial. Usualmente, los mapas se despliegan con electrogramas bipolares pico a pico de 15 a $20 \mathrm{~mm}^{29}$.

Durante la taquicardia ventricular, la presencia de potenciales presistólicos y diastólicos usualmente representa activación local y mediante la técnica de encarrilamiento o entrainment pueden definirse como vías críticas de circuito ${ }^{29}$. En pacientes con taquicardia ventricular con hemodinámica inestable se ha empleado la técnica del mapeo de la o las cicatrices en ritmo sinusal y la realización de ablación guiada por este sustrato ${ }^{30}$. 


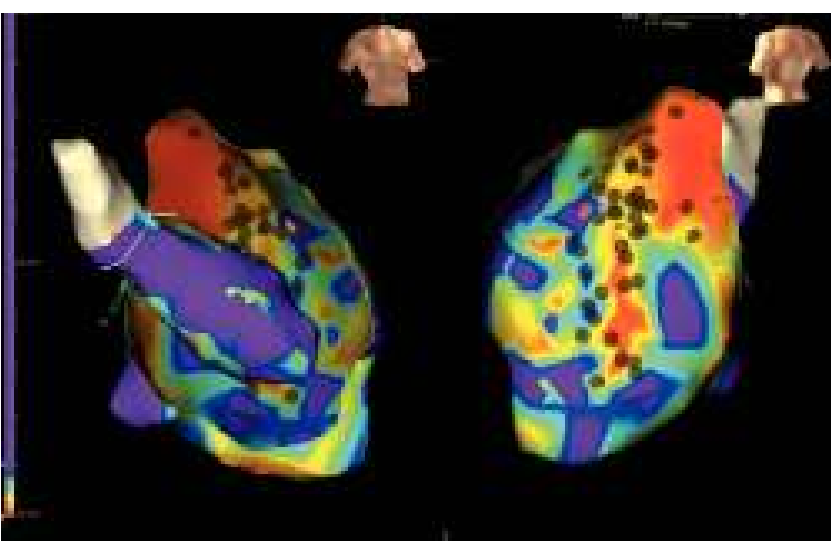

Figura 1 Mapa de voltaje epicárdico del ventrículo izquierdo en un paciente con cardiopatía chagásica, en donde se aprecian cicatrices extensas (áreas en rojo). El área púrpura es tejido sano y los demás colores representan la zona de transición entre el tejido sano y la cicatriz eléctrica.

La realización del mapa ventricular puede mejorarse mediante sistemas de imágenes. Así mismo, un estudio reciente demostró la factibilidad de combinar mapeo electroanatómico mediante sistema CARTO con tomografía axial computarizada (TAC) de corazón y arterias coronarias en pacientes con taquicardia ventricular y cardiopatía chagásica ${ }^{31}$. Los autores encontraron, además, que las anormalidades del movimiento de la pared en la TAC se correlacionaban con cicatrices transmurales en el mapeo electroanatómico, aunque se encontraban más cicatrices con el mapeo que con la TAC. La visualización del árbol coronario es útil para disminuir la probabilidad de causar una lesión coronaria durante las aplicaciones de radiofrecuencia en la zona epicárdica.

Los estudios que han evaluado sistemáticamente el endocardio y el epicardio con mapeo electroanatómico concluyen que en cardiopatía chagásica las cicatrices son mayores en el epicardio. Adicionalmente, la ablación endoepicárdica reduce el número de descargas en pacientes portadores de cardiodesfibriladores ${ }^{29}$. En este trabajo también se encontró que la mayor duración del electrograma bipolar en el endocardio se correlacionaba con cicatrices en el epicardio. Sin embargo, y a pesar de todas las técnicas anteriores, en el $16 \%$ de los pacientes no pudieron ser eliminadas las taquicardias ventriculares. En el seguimiento a un año, el 78\% permaneció libre de arritmia ventricular.

De otro lado, no hay estudios prospectivos aleatorizados que comparen la ablación de taquicardia ventricular con o sin mapeo tridimensional, pero dada la necesidad de definición del sustrato, de utilizar diferentes técnicas de ablación (decanalización, homogenización de la cicatriz, ablación sobre istmos críticos, entre otros), definitivamente se necesita del mapeo tridimensional para obtener un resultado más certero.

\section{Recomendaciones}

\section{Clase I}

- En pacientes con cardiopatía chagásica que serán llevados a ablación por presencia de taquicardia ventricular se recomienda el uso de mapeo tridimensional para un análisis adecuado del sustrato y mejor definición de los sitios de ablación (nivel de evidencia C).

\section{Bibliografía}

1. Chagas disease in Latin America: an epidemiological update based on 2010 estimates. Wkly Epidemiol Rec. 2015;90:33-43.

2. Malik LH, Singh GD, Amsterdam EA. The Epidemiology, Clinical Manifestations, and Management of Chagas Heart Disease. Clin Cardiol. 2015;38:565-9.

3. Organización Panamericana de la Salud. Estimación cuantitativa de la enfermedad de Chagas en las Américas. Montevideo, Uruguay: Organización Panamericana de la Salud; 2006.

4. PAHO. Health Conditions in the Americas. Volume I. Washington: Pan American Health Organization; 1990.

5. Schofield CJ, Dias JC. A cost-benefit analysis of Chagas disease control. Mem Inst Oswaldo Cruz. 1991;86:285-2.

6. Tovar NC, Echeverry MC, Mora G. Presencia de anticuerpos contra neurorreceptores cardiacos de acetilcolina muscarínicos tipo II en pacientes con enfermedad de Chagas e implantación de marcapasos. Biomedica 2009;29:476-84.

7. Rassi Jr A, Rassi A, Marin-Neto JA. Chagas heart disease: pathophysiologic mechanisms, prognostic factors and risk stratification. Mem Inst Oswaldo Cruz. 2009;104 Suppl 1:152-8.

8. Traina M, Sanchez D, Hernandez S. Chagasic cardiomyopathy is associated with increased morbidity and mortality compared with nonischemic cardiomyopathy among Latin American immigrants in Los Angeles. Circulation. 2012;126:A18171.

9. Mora G. Arritmias ventriculares y supraventriculares en la enfermedad de Chagas. En: Rosas F, Vanegas D. Enfermedad de Chagas. Bogotá: Panamericana formas e impresos; 2007. p. 89-93.

10. Lopes ER, Tafuri WL. Involvement of the autonomic nervous system in Chagas heart disease. Rev Soc Bras Med Trop. 1983; 16:206-11.

11. Miranda $\mathrm{CH}$, Figueiredo $\mathrm{AB}$, Maciel BC. Sustained ventricular tachycardia is associated with regional myocardial sympathetic denervation assessed with 123I-metaiodobenzylguanidine in chronic Chagas cardiomyopathy. J Nucl Med. 2011;52:50410.

12. Carrasco HA, Guerrero L, Parada H. Ventricular arrhythmias and left ventricular myocardial function in chronic chagasic patients. Int J Cardiol. 1990;28:35-41.

13. Healy C, Viles-Gonzalez JF, Sáenz LC, Soto M, Ramírez JD, d'Avila A, Arrhythmias in chagasic cardiomyopathy. Card Electrophysiol Clin. 2015;7:251-68.

14. Manzullo EC, Chuit R. Risk of death due to chronic chagasic cardiopathy. Mem Inst Oswaldo Cruz. 1999;94 Suppl 1:317-20.

15. Bestetti RB, Freitas OC, Muccillo G. Clinical and morphological characteristics associated with sudden cardiac death in patients with Chagas' disease. Eur Heart J. 1993;14:1610-4.

16. Piccini JP, Berger JS, O'Connor CM. Amiodarone for the prevention of sudden cardiac death: a meta-analysis of randomized controlled trials. Eur Heart J. 2009;30:1245-53.

17. Tomaz MP, Lopes A, Da Costa MO, Pinho AL. Ventricular arrhythmia in Chagas disease. Rev Soc Bras Med Trop. 2015;48:4-10.

18. Muratore CA, Batista Sa LA, Chilae PA, et al. Implantable cardioverter defibrillatiors and Chagas' disease: results of the ICD Registry Latin America. Europace. 2009;11:164-8.

19. Gali W, Sarabanda A, Baggio A. Implantable cardioverter defibrillators for treatment of sustained ventricular arrhythmias in patients with Chagas' heart disease: comparison with a control group treated with amiodarone alone. Europace. 2014;16:674-80.

20. Mora G, Echevarry M, Rey G, López C, Posada L, Rivas F. Frecuencia de anticuerpos anti-Trypanosoma cruzi en pacientes 
portadores de marcapasos de la Clínica San Pedro Claver de Bogotá. Biomédica. 2007;27:483-9.

21. Schron EB, Exner DV, Yao Q, Jenkins LS, Steinberg JS, Cook JR, et al. Quality of life in the antiarrhythmics versus implantable defibrillators trial: impact of therapy and influence of adverse symptoms and defibrillator shocks. Circulation. 2002;105:58994.

22. Sarabanda AV, Sosa E, Simões MV, Figueiredo GL, Pintya AO, Marin-Neto JA. Ventricular tachycardia in Chagas' disease: a comparison of clinical, angiographic, electrophysiologic and myocardial perfusion disturbances between patients presenting with either sustained or nonsustained forms. Int J Cardiol. 2005;102:9-19.

23. Della Bella P, De Ponti R, Uriarte JA, Tondo C, Klersy C, Carbucicchio $\mathrm{C}$, et al. Catheter ablation and antiarrhythmic drugs for haemodynamically tolerated post-infarction ventricular tachycardia. Long-term outcome in relation to acute electrophysiological findings. Eur Heart J. 2002;23:414-24.

24. Tomaz Barbosa MP, Lopes do Carmo AA, Da Costa Rocha MO, Pinho Ribeiro AL. Ventricular arrhythmias in Chagas disease. Rev Soc Bras Med Trop. 2015;48:4-10.

25. Scanavacca $M$. epicardial ablation for ventricular tachycardia in chronic Chagas heart disease. Arq Bras Cardiol. 2014;102:524-8.
26. Sosa E, Scanavacca M, D'Avila A, et al. Radiofrequency catheter ablation of ventricular tachycardia guided by nonsurgical epicardial mapping in chronic chagasic heart disease. PACE. 1999;22:128-30.

27. Sosa E, Scanavacca M, D'Avila A, et al. A new technique to perform epicardial mapping in the electrophysiology laboratory J Cardiovasc Electrophysiol. 1996;7:531-6.

28. D'Avila A, Scanavacca M, Sosa E, et al. Pericardial anatomy for the interventional electrophysiologist. J Cardiovasc Electrophysiol. 2003;14:422-30.

29. Henz BD, Nascimiento T, Dietrich C, et al. Simultaneous epicardial and endocardial substracte mapping and radiofrequency catheter ablation as first-line treatment for ventricular tachycardia and frequent ICD shocks in chronic chagasic cardiomyopathy. J Interv Card Electrophysiol. 2009;26:195205.

30. Tomaz MP, Lopes A, Da Costa M, Phino A. Ventricular arrhythmias in chagas disease. Rev Soc Bras Med Trop. 2015;48:4-10.

31. Valdigem BP, Da Silva NJ, Dietrich CO, Moreira D, Sasdelli R, Pinto IM, et al. Accuracy of epicardial electroanatomic mapping and ablation of sustained ventricular tachycardia merged with heart CT scan in chronic chagasic cardiomyopathy. J Interv Card Electrophysiol. 2010;29:119-25. 